\title{
Impact Analysis of PM magnetization level on motor performance: simulations and experimental results
}

\author{
Tiago Staudt, Thiago Akinaga, \\ EMBRACO R\&D Department, tiago.staudt@embraco.com, thiago.h.akinaga@embraco.com \\ Leonardo Ulian Lopes, \\ UFSC - Engineering Department, Campus Blumenau, leonardo.ulian@ufsc.br \\ Fernando Maccari, Paulo A. P. Wendhausen \\ UFSC-Mechanical Engineering Department, fernando.maccari@yahoo.com.br, paulo.wendhausen@ufsc.br
}

\begin{abstract}
This paper addresses the impact of magnetization level tolerances in ferrite-based permanent magnets (PM) on the performance of brushless DC motors. A case study is prepared using three motor prototypes assembled with controlled samples of ferrite arc segments, in terms of magnetization. The investigation is performed both experimentally and through simulations using the software SPEED. The results show that narrow tolerance margins for the magnetization conditions in the production line can potentially minimize the use of raw material on such motors whereas keeping the efficiency and torque behavior required by the application.
\end{abstract}

Index Terms - brushless DC motors; machine testing; magnetization; ferrite magnets; quality assurance; quality control; magnetization quality assessment.

\section{THE DEPENDENCE OF MOTOR PERFORMANCE ON PM MAGNETIZATION CONDITIONS}

Manufacturing tolerances are an inherent and inevitable characteristic of any production line. From a manufacturing process point of view, the experience shows that the smaller the tolerances, the more expensive it is to manufacture an item in terms of equipments and testing capabilities to assure small deviations among the products. From the product's side, the designer has to guarantee at least the minimum performance level required by the application and a worst-case scenario design usually applies to always fulfill the requirements. This fact may lead to non-optimal use of raw materials and extra costs for the product. A potentially better solution is usually a trade-off among process and product requirements. Many papers in the literature discuss magnetization characteristics of permanent magnet motors, e.g. [1]-[4]. To address this issue, this paper investigates the tolerances of one key aspect on a ferrite PM-based motor design: the influence PM magnetization conditions on motor's performance and raw material utilization (copper and steel).

The paper proposes an experimental investigation on the dependence of several motor parameters that depends upon PM magnetization levels. A surface mounted PM-ferrite, brushless DC motor (BLDC) with $200 \mathrm{~W}$ rated power is used in this case study to assess the differences in performance due to different PM magnetization conditions. Fig. 1 shows the motor topology with 6 stator slots and 4 rotor poles. 


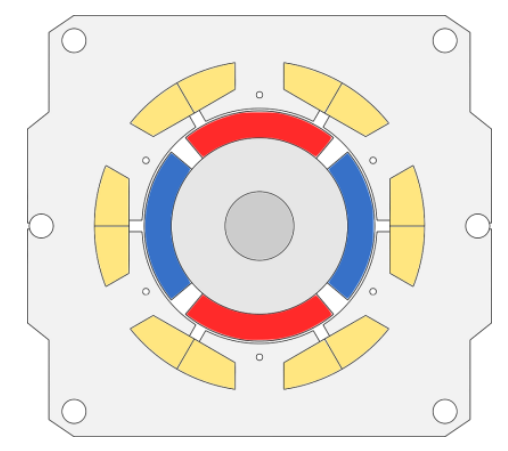

Fig. 1 - Cross-sectional view of the BLDC 6 slots 4 poles motor topology used in the case study.

Details about the electromagnetic design of such motor can be found in [5]. The first part of the paper presents the methodology used to measure the PM polarization before their assemblies to the rotor. Then, experimental tests are reported, comparing performance characteristics among different rotors. Finally, a discussion on raw materials utilization is presented based on a simulation approach.

\section{METHODOLOGY}

A. Assessing the magnetization conditions: the use of Helmholtz coil for measuring the magnetization level of PM samples

The arc segments used in the BLDC have been magnetized and their polarizations have been individually measured by means of a Helmholtz coil. Fig. 2 shows the Helmholtz coil used for the measurements [6].

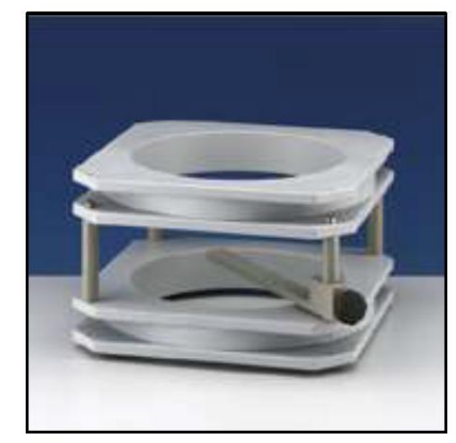

Fig. 2 - Helmholtz coil from Brockhauss Messtechnik used in the tests.

The magnetic material characterization using the Helmholtz coil evaluates the magnetic properties of permanent magnets in open-circuit conditions, without applying external magnetic fields to the samples, hence not influencing their magnetization state. The following properties are measured using this device at room temperature:

- Magnetic Flux (V.s).

- Magnetic Polarization at the working point (open-circuit) (T).

For the measurements on this device, the following considerations must be taken into account:

- The PM samples must be in the magnetized state.

- They must be lower than 50,0 $\mathrm{mm}$ in any direction (for the considered coil).

- The sample volume must be known in order to determine the magnetic polarization. 
- The magnetization axis must be known.

Several groups of ferrite arc segments have been magnetized using different devices and conditions (A, B, C, D and E). Fig. 3 shows the dispersion of the results for the magnetic flux and polarization for each group. Differences in the mean value and standard deviation among the groups can be noticed and, from these results, best and the worst magnetized samples (in terms of magnetic flux) have been selected to be used in the tests presented in the sequence.

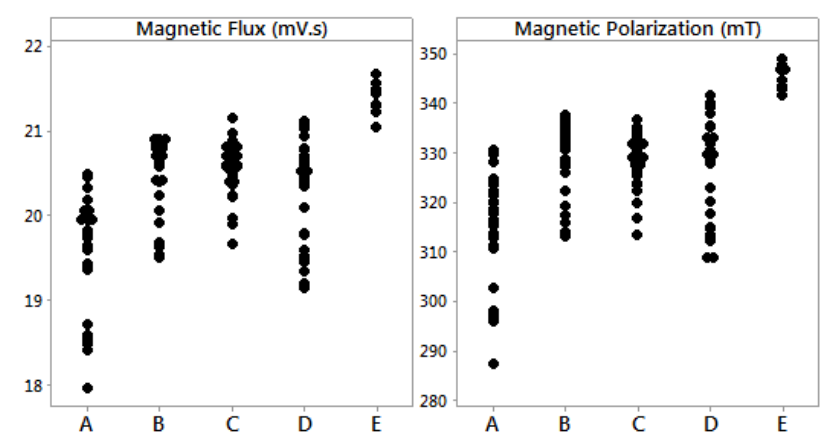

Fig. 3 - Magnetic flux and polarization measured using the Helmholtz coil for ferrite arc segments.

\section{B. Methodology applied to evaluate the impact of PM magnetization tolerances on motor performance}

The approach used to analyze the impact of PM magnetization in motor performance seeks to isolate their effect from other manufacturing tolerances. Therefore, the same stator has been used for all tests.

Regarding the rotor, four arc segments of alternate polarities (NSNS) are required for each assembly. The rotor has been prepared with the core produced with silicon-iron steel. The rotor stacks have been prepared from the same batch and in the same day. The selected PM's from Fig. 3 have being separated in two groups: the ones with highest values of polarization and the ones with the lowest levels. Then, this controlled PM arc segments have been assembled to the rotor stacks to evaluate the effect of different magnetization levels in motor performance. Fig. 4 shows the three rotor samples that have been prepared.

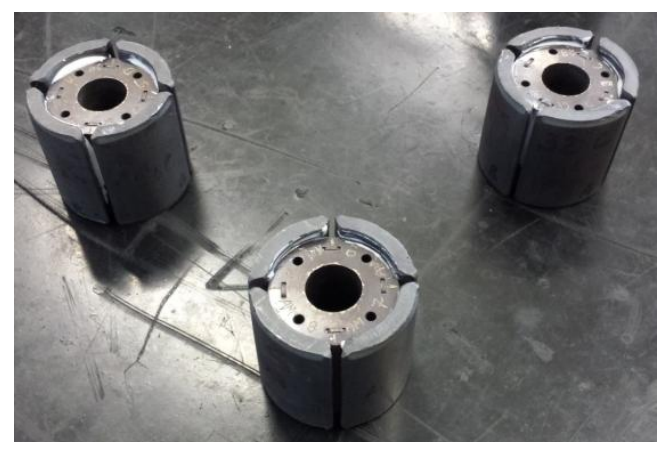

Fig. 4 - Picture of the rotor samples under analysis.

Table I summarizes the average magnetization levels of the PM arc segments in terms of polarization and flux that have been chosen to assemble each rotor. The average polarization of $\mathrm{R} 1$ is $348.2 \mathrm{mT}$ and this value is assumed to be the reference. The average is calculated by considering the polarization of each PM individually. Then, R2 rotor is assembled with 4 arc segments resulting in an 
average polarization that is $12.7 \%$ below the reference. Sample R3 is $2.7 \%$ below the reference. This nomenclature is used throughout this paper to designate the different rotors under analysis.

TABLE I - AVERAGE MAGNETIZATION FOR THE THREE ROTOR SAMPLES CONSIDERED IN THIS REPORT.

\begin{tabular}{|c|c|c|c|c|}
\hline & Average Polarization [mT] & $\%$ Difference & Average Flux [mV.s] & $\%$ Difference \\
\hline $\mathrm{R} 1$ & 348,2 & REF & 21,5 & REF \\
\hline $\mathrm{R} 2$ & 304,1 & $-12,7 \%$ & 18,9 & $-12,3 \%$ \\
\hline R3 & 338,8 & $-2,7 \%$ & 21,1 & $-2,1 \%$ \\
\hline
\end{tabular}

It is important to mention that the polarization levels indicated in Table 1 are measured in opencircuit using the Helmholtz coil and do not represent directly the PM's remanence, which is the polarization in the absence of self-demagnetizing fields. For the simulation considered latter in this paper, the actual values of $\mathrm{Br}$ have been estimated by using the software SPEED [7] by adjusting the Br coefficient to match the measured and simulated back-emf waveforms

\section{EXPERIMENTAL TESTS FOR THE IMPACT ANALYSIS OF PM MAGNETIZATION STATE ON MOTOR'S PERFORMANCE}

The following tests have been performed considering a winding temperature of $30{ }^{\circ} \mathrm{C}+-5^{\circ} \mathrm{C}$ :

- Back-EMF.

- Torque versus Current.

- Efficiency versus Torque.

- Maximum torque versus speed.

The experimental results are also used to calibrate the simulation model developed in the software SPEED version 10.02. Once calibrated, the SPEED model is used to estimate the impact of PM magnetization conditions in raw material utilization for obtaining about the same performance.

\section{A. Back-EMF}

1) Back-EMF at 2000 rpm

The measured back-EMF curves at $2000 \mathrm{rpm}$ for the three rotors are shown in Fig. 5.

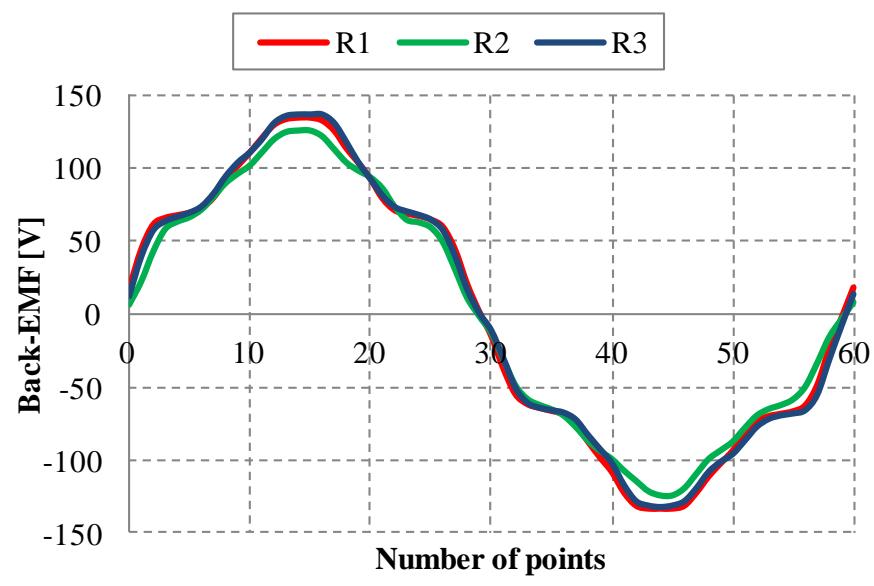

Fig. 5 - Measured back-EMF for the 3 rotor samples at $2000 \mathrm{rpm}$.

To better evaluate the results, Fig. 6 shows a zoom in the region of interest for the 3 distinct rotors 
at $2000 \mathrm{rpm}$.

It can be remarked that the samples R1 and R3 have similar waveforms. This is expected, since their polarizations $(\mathrm{P})$ are similar (2.7\% difference). The R2 sample has roughly $12.7 \%$ less flux than the R1 sample, which represent a peak value in the back-EMF waveform that is $6 \%$ lower.

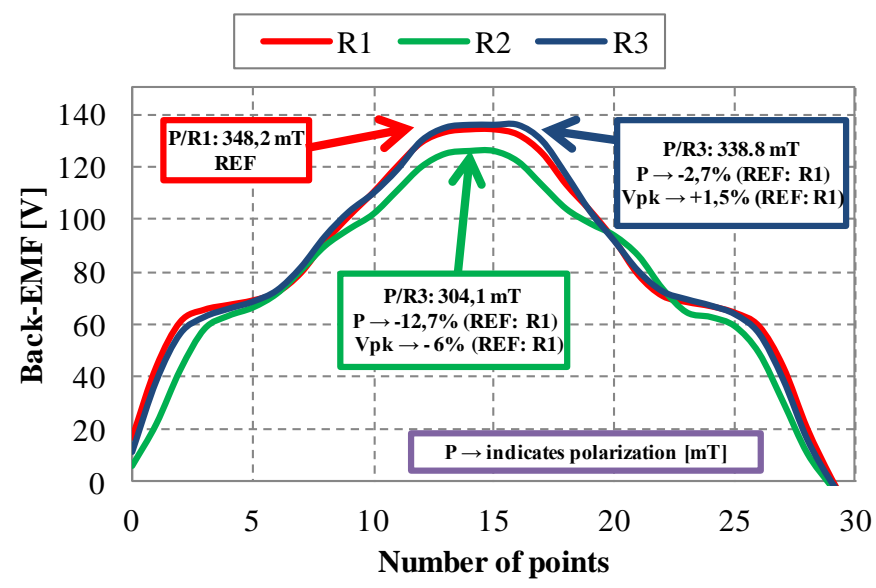

Fig. 6 - Measured Back-EMF (zoomed) for the 3 rotor versions at $2000 \mathrm{rpm}$.

2) Measured Back-EMF at 3000 rpm

Similar behavior as identified in Fig. 6 can be verified in Fig. 7 for a zoomed region at $3000 \mathrm{rpm}$ for the three samples. While the magnetization level is $12.7 \%$ lower in R2 sample, the peak difference is $6.5 \%$ lower in this sample than it is in the R1.

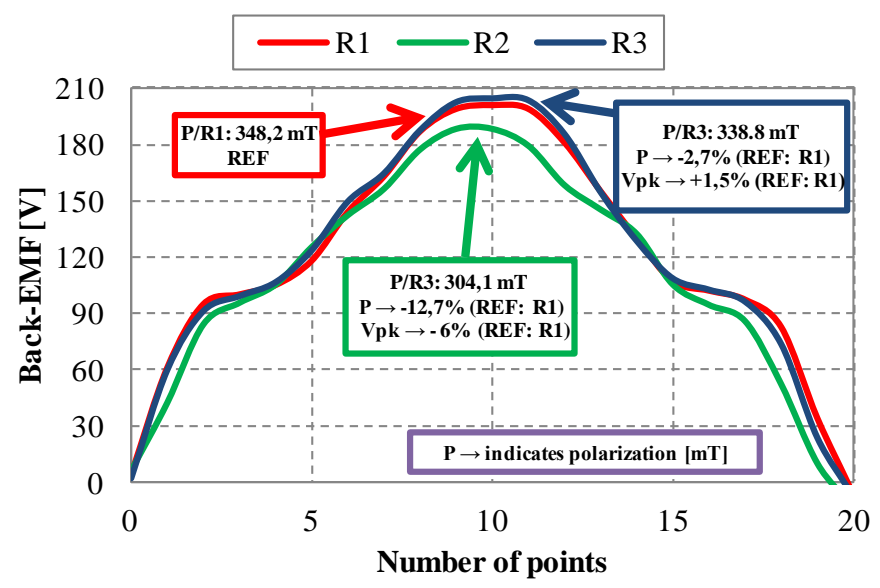

Fig. 7 - Measured Back-EMF (zoomed) for the 3 rotor versions at $3000 \mathrm{rpm}$.

B. Torque versus Current at $2000 \mathrm{rpm}$

The torque is proportional to current and is given by [1]:

$$
T_{e m}=k_{T} I
$$

where $\mathrm{k}_{\mathrm{T}}$ is the torque constant.

By plotting a torque versus current one can roughly determine the $\mathrm{k}_{\mathrm{T}}$ constant for different machines. In this case, since the stator is the same in all the tests, the PM magnetization level is the only variable that changes in each rotor. Fig. 8 shows the results from 0.2 up to $0.6 \mathrm{~A}$, which approximately represents a variation from $1 \mathrm{up}$ to $4 \mathrm{kgf.cm}$ in the electromagnetic torque. 
To estimate the $\mathrm{k}_{\mathrm{T}}$ coefficient for all different rotors, a linear trend line is calculated and the results are shown in Fig. 8. The R1 sample gives an angular coefficient of $\mathrm{k}_{\mathrm{T}_{\mathrm{R} 1}}=6.5709$, similar to the one of $\mathrm{R} 3$ sample as expected, which gives $\mathrm{k}_{\mathrm{T}_{\mathrm{R} 3}}=6.5339$. Regarding the $\mathrm{R} 2$ " sample, the $\mathrm{k}_{\mathrm{T}_{\mathrm{R} 2}}=$ 6.0972. The factor $\frac{\mathrm{k}_{\mathrm{T}_{\mathrm{R} 2}}}{\mathrm{k}_{\mathrm{T}_{\mathrm{R} 1}}}=\frac{6.0972}{6.5709}=0,928$ indicates that the $\mathrm{R} 2$ motor can provides roughly $7,2 \%$ less torque than the $\mathrm{R} 1$ one.

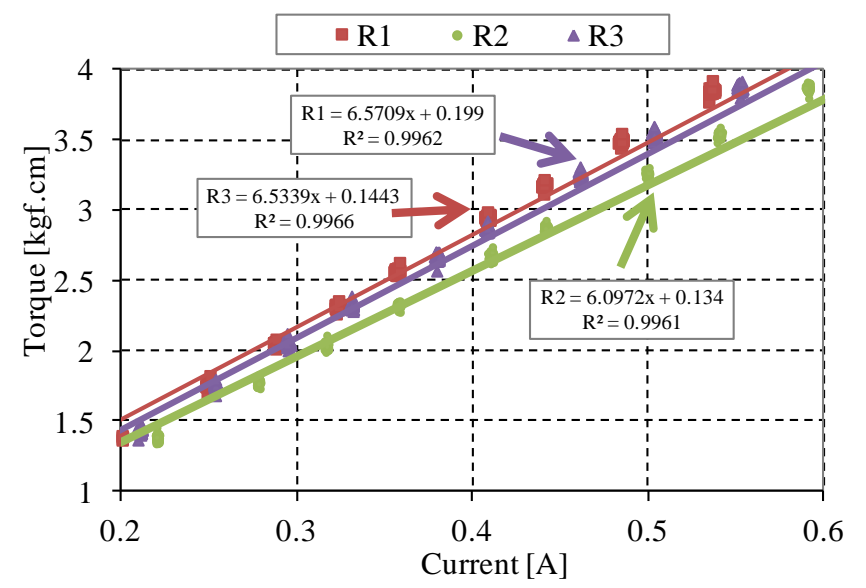

Fig. 8 - Torque versus current plot at $2000 \mathrm{rpm}$ : trend lines to obtain coefficient $k_{T}$.

\section{Efficiency versus Torque curves}

Efficiency is one of the most fundamental parameter in motor design. This section investigates the influence of PM magnetization in this aspect in terms of the produced electromagnetic torque through experimental tests. To have a clearer overview over the entire torque range, the efficiency is plotted in terms of the torque for $2000 \mathrm{rpm}, 1600 \mathrm{rpm}$ and $3000 \mathrm{rpm}$ for the 3 rotor assemblies.

1) Efficiency versus Torque at $2000 \mathrm{rpm}$

The first comparison is shown at $2000 \mathrm{rpm}$ in Fig. 9. It is possible to infer that for lower excitation (around $1 \mathrm{kgf.cm}$ ), the iron losses dominate the efficiency curve and the R2 rotor is more efficient since it has a lower Br. At $2 \mathrm{kgf.cm}$, the efficiency curve reaches its peak with similar efficiency for all rotors. At this point, the iron losses and copper losses are nearly equivalent. From this point on, the copper losses dominates the response, since more current is supplied to the motor to sustain the required torque. Consequently, the losses are higher for the R2 rotor because of the lower induced back-emf (for the same torque, more current - and winding losses - are required for the R2 rotor). In the worst analyzed case (4.5 kgf.cm), the efficiency is about $1.5 \%$ lower for the R2 than it is for the $\mathrm{R} 1$ rotor. 


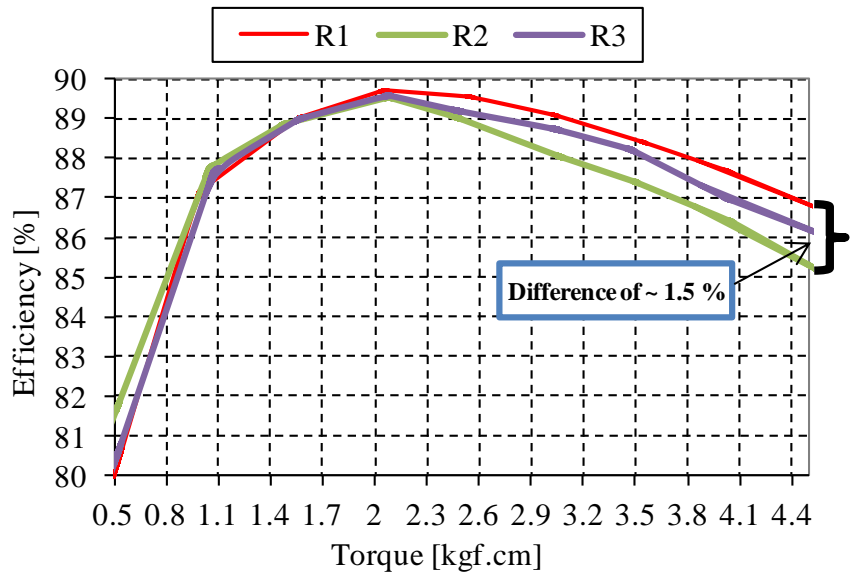

Fig. 9 - Efficiency versus torque curve at $2000 \mathrm{rpm}$.

Efficiency versus Torque at $1600 \mathrm{rpm}$ and $3000 \mathrm{rpm}$ (measured only)

Similar behavior as notice for the $2000 \mathrm{rpm}$ for the efficiency curve can be remarked at 1600 and 3000 rpm as shown in Fig. 10 (1600 rpm) and Fig. 11 (3000 rpm).

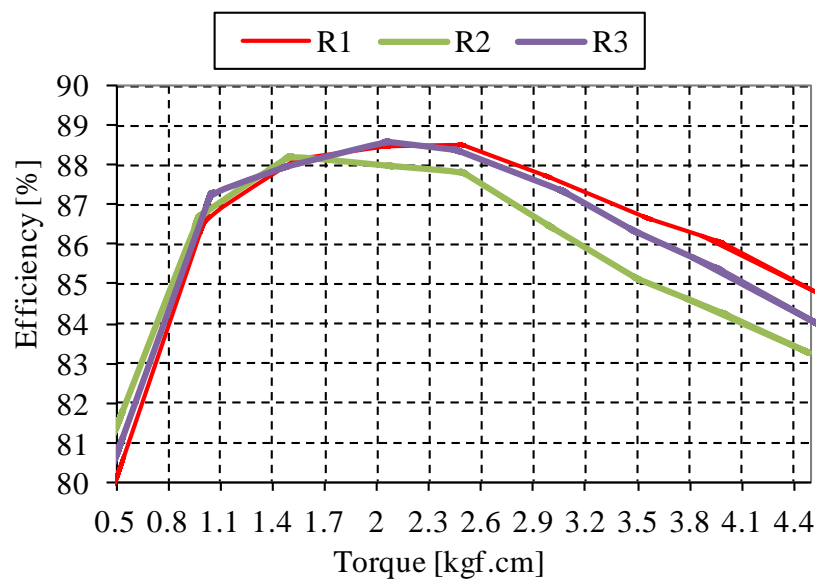

Fig. 10 - Efficiency versus torque curve at $1600 \mathrm{rpm}$.

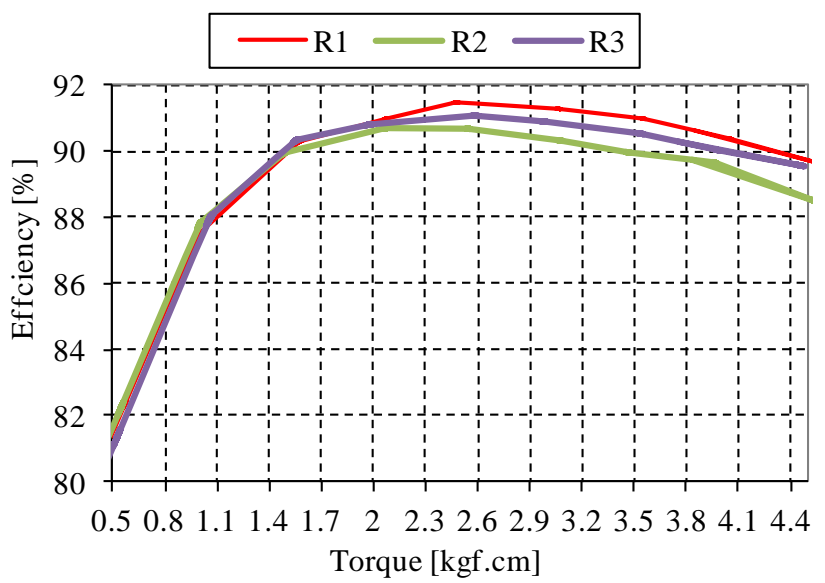

Fig. 11 - Efficiency versus torque curve at $3000 \mathrm{rpm}$.

D. Maximum torque output versus speed

The maximum torque versus speed curve is plotted by feeding the motor through the inverter with rated current while the speed is varied from $4500 \mathrm{rpm}$ up to $0 \mathrm{rpm}$. Fig. 12 shows the measured 
results.

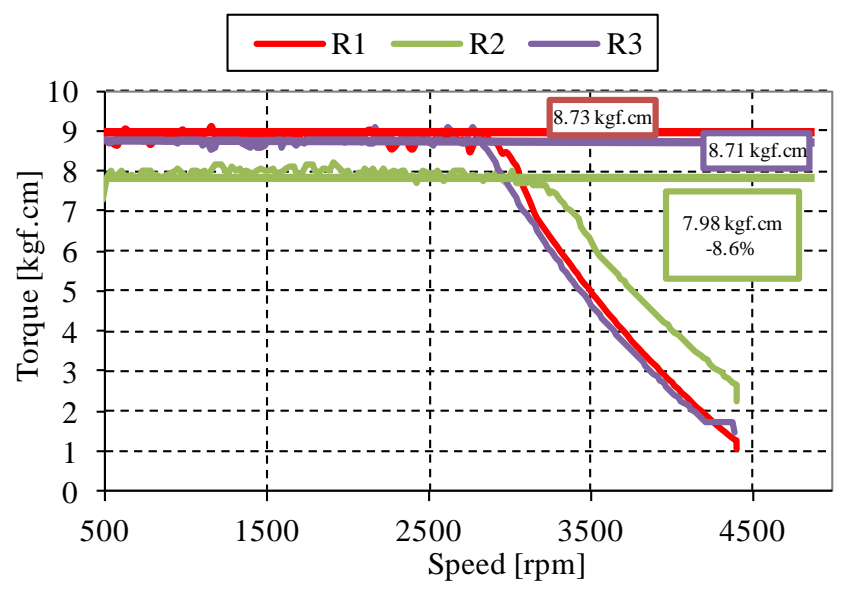

Fig. 12 - Measured maximum torque versus speed curve.

It is possible to remark that the $\mathrm{R} 2$ rotor provides $8.6 \%$ less torque than the $\mathrm{R} 1$ and $\mathrm{R} 3$ rotors. This result confirms the one inferred from the torque coefficient analysis $\left(\mathrm{k}_{\mathrm{T}}\right)$, which indicated $7.2 \%$ less torque in the R2 motor.

\section{DISCUSSION ON THE IMPACT OVER PERFORMANCE AND RAW MATERIAL UTILIZATION}

In order to assess the impact of PM magnetization in raw material utilization, this section presents a case study as follows. Assuming that the R2 rotor satisfies the application requirements in terms of torque and efficiency, the stator of R1 rotor is modified so that it has in the final design the same outcomes of R2 motor. In other words, the extra raw material of R1 rotor is eliminated so that we can take benefit of a fully saturated PM as the one in rotor R1. This investigation is made by using a calibrated SPEED model considering the experimental machine presented in this paper.

\section{A. Calibration of SPEED software}

In order to calibrate the SPEED software for the comparisons that follows, the experimental results are confronted to the ones obtained from simulation in SPEED. The PM remanence is adjusted in SPEED in order to obtain a back-EMF waveform that matches the peak of the measured one. Fig. 13 shows a comparison of the R1 sample and the results obtained in SPEED using the Br value of 366 $\mathrm{mT}$. It can be seen that this $\mathrm{Br}$ results in a waveform that matches the experimental one with high accuracy. 


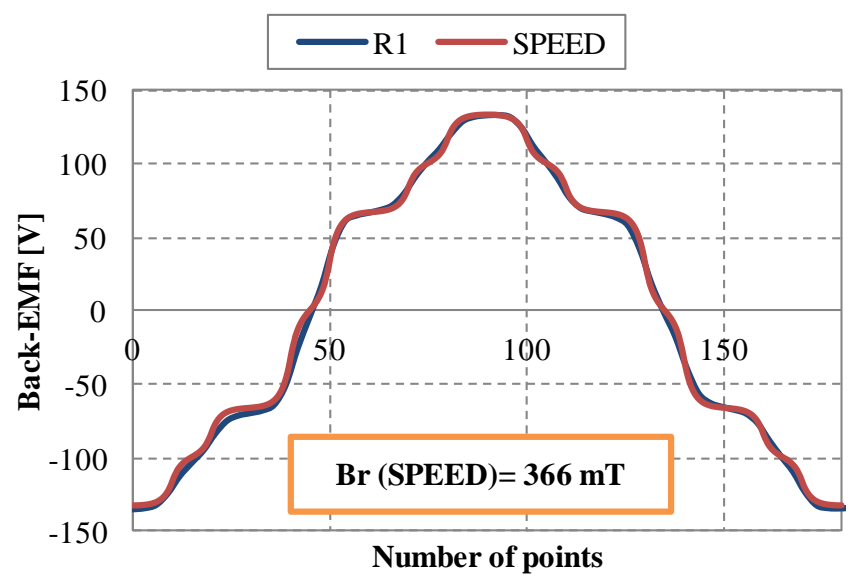

Fig. 13 - Comparison among experimental and simulation results for the BACK-EMF at $2000 \mathrm{rpm}$ and sample R1.

Similarly, the same approach is used for matching the R2 and R3 waveforms with the simulation from SPEED. Fig. 14 depicts the waveform comparison for R2 sample. A $\mathrm{Br}=346 \mathrm{mT}$ provides a good agreement among the curves.

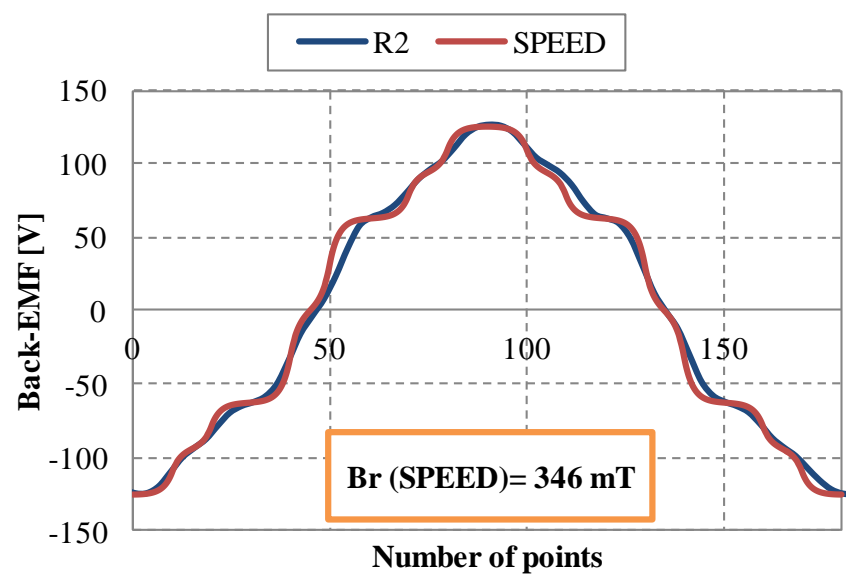

Fig. 14 - Comparison among experimental and simulation results for the BACK-EMF at $2000 \mathrm{rpm}$ and sample R2.

B. Torque versus speed curve: adjusting the number of turns

Fig. 15 shows the original simulated curves of R1 and R2 rotors performance in terms of torque and speed. It indicates that it is possible to reduce the R1 torque in about 5.5\% in order to get similar results. Fig. 15 also shows a first attempt to reduce raw material utilization by reducing the number of turns by $13 \%$. The "NEW R1 temp" motor now has the same torque output as R2 with less turns. 


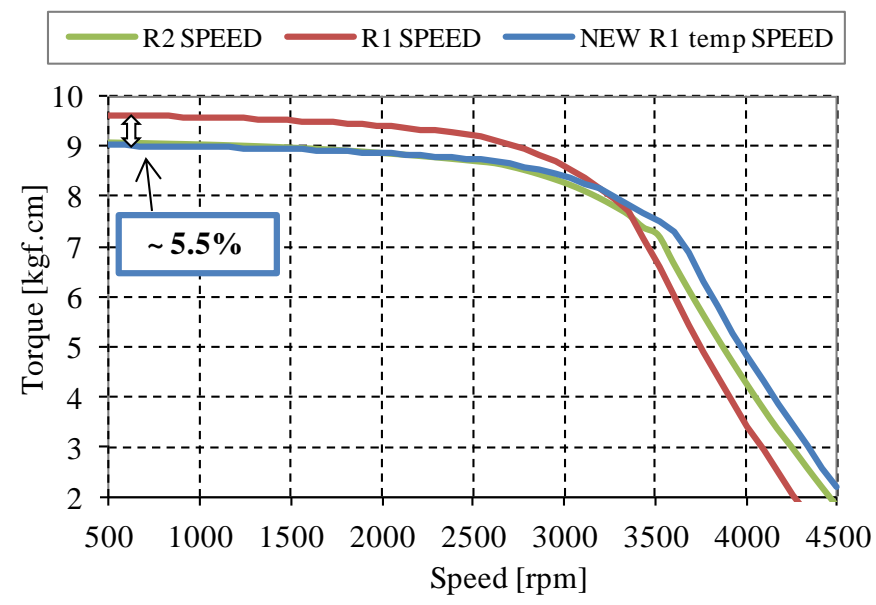

Fig. 15 - Original differences for R1 and R2 rotors and the new R1 motor with reduced number of turns.

C. Efficiency versus torque curve: reducing the wire diameter to match efficiency

The original efficiency curves for R1 and R2 rotors are compared to the efficiency of the "NEW R1 temp" motor without any other change besides the number of turns. The results are shown in Fig. 16.

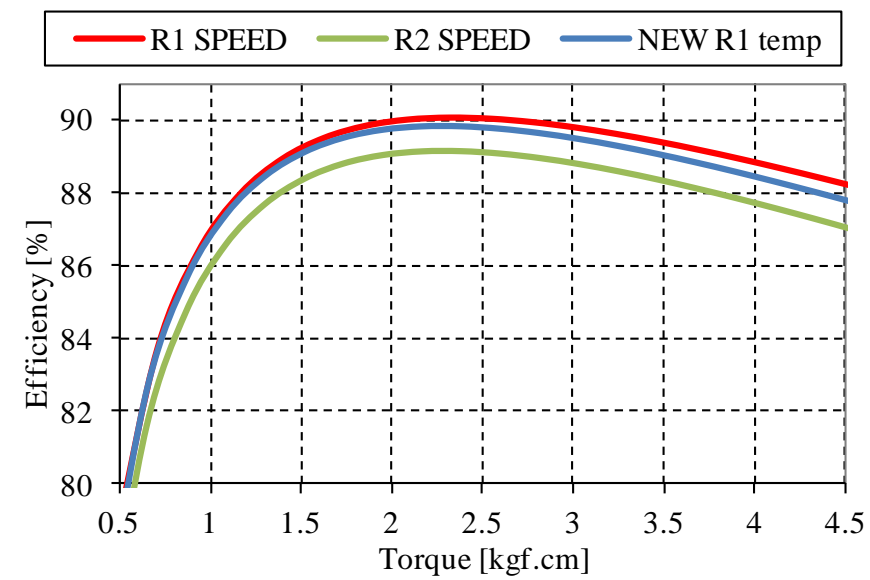

Fig. 16 - Original efficiency versus torque curve at $2000 \mathrm{rpm}$ compared to the new motor.

It is noticed that that the efficiency of the "NEW R1 temp" motor is closer to the R2, but we still have a margin to work with. Therefore, the wire diameter is reduced by $6.6 \%$ in order to gain in copper weight aiming to match R2 efficiency (NEW R1). Fig. 17 depicts the results. It is possible to remark that these design modifications allow redesigning the R1 motor so that it nearly matches the performance of the R2 motor in terms of torque production and efficiency. 


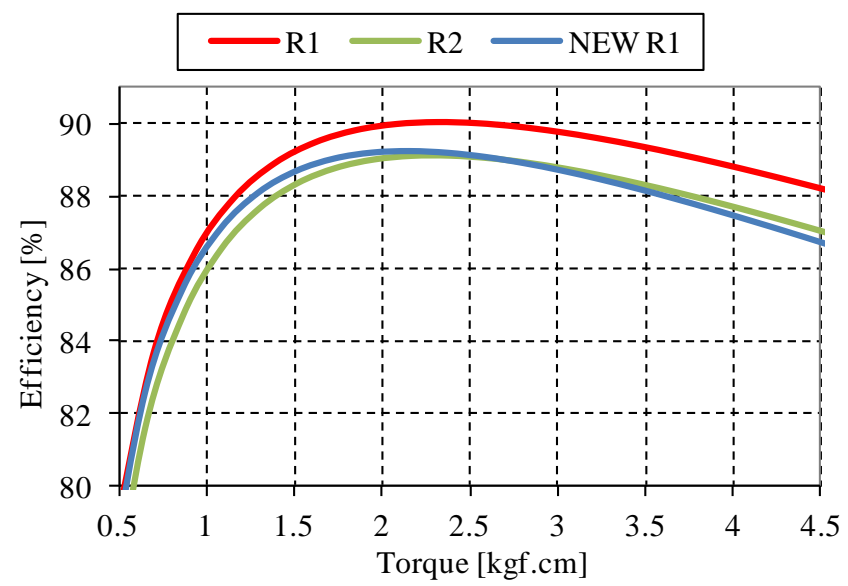

Fig. 17 - Effect of reducing the number of turns and wire diameter for the final design NEW R1.

Table II summarizes the impact on raw materials. There is a decrease of nearly $21 \%$ in cooper weight by reducing the number of turns and the wire diameter considering narrower tolerances in the PM arc segments magnetization conditions.

TABLE II -IMPACT ON RAW MATERIAL UTILIZATION.

\begin{tabular}{cc}
\hline & Copper weight $[\mathrm{kg}]$ \\
\hline R1 / R2 & 0.3109 \\
NEW R1 & 0.2457 \\
Diff \% & $-20.97 \%$ \\
\hline
\end{tabular}

\section{CONCLUSIONS}

This paper addresses the impact of manufacturing tolerances in the magnetization level of ferrite arc segments on BLDC motor design. The effect is analyzed from a performance point of view and the best utilization of raw material is discussed using both experimental and simulation results. It has been found that, for the considered case study, if one can reduce the tolerance margins for magnetization of ferrite arc segments in the manufacturing line, a significant amount of raw material can be reduced from the final motor design for the same application requirements. In the considered case study, an improvement of $12 \%$ resulted in a copper reduction of $21 \%$.

\section{REFERENCES}

[1] K. W. Lee, J. Hong, S. B. Lee and S. Lee, "Quality Assurance Testing for Magnetization Quality Assessment of BLDC Motors Used in Compressors," in IEEE Transactions on Industry Applications, vol. 46, no. 6, pp. 2452-2458, Nov.-Dec. 2010.

[2] M. F. Hsieh, D. G. Dorrell, C. K. Lin, P. T. Chen and P. Y. P. Wung, "Modeling and Effects of In Situ Magnetization of Isotropic Ferrite Magnet Motors," in IEEE Transactions on Industry Applications, vol. 50, no. 1, pp. 364-374, Jan.-Feb. 2014.

[3] M. Hsieh and S. Yu, "In-situ magnetization of permanent magnet machines considering magnetizer capacity and connection types," 2015 IEEE Magnetics Conference (INTERMAG), Beijing, 2015, pp. 1-1.

[4] M. F. Hsieh, Y. C. Hsu and P. T. Chen, "Analysis and Experimental Study of Permanent Magnet Machines With InSitu Magnetization," in IEEE Transactions on Magnetics, vol. 49, no. 5, pp. 2351-2354, May 2013.

[5] Hendershot, JR., Miller, TJE; Desing of Brushless Permanent-Magnet Machines. ISBN 978-0-9840687-0-8. 2010.

[6] Brockhaus Messtechnik Website. Accessed on 20/04/2015: http://brockhaus.com/de/messtechnik/messtechnik-fuerhartmagnetische-werkstoffe/spulen/helmholtzspule/

[7] Miller, TJE, MI McGilp. SPEED PC-BDC 10.02. SPEED laboratory, Glasgow. Computational Dynamic Ltd., 2015. 